\title{
REAL ZEROS OF HOLOMORPHIC HECKE CUSP FORMS AND SIEVING SHORT INTERVALS
}

\author{
KAISA MATOMÄKI
}

\begin{abstract}
We study so-called real zeros of holomorphic Hecke cusp forms, that is zeros on three geodesic segments on which the cusp form (or a multiple of it) takes real values. Ghosh and Sarnak, who were the first to study this problem, showed that existence of many such zeros follows if many short intervals contain numbers whose all prime factors belong to a certain subset of the primes. We prove new results concerning this sieving problem which leads to improved lower bounds for the number of real zeros.
\end{abstract}

\section{INTRODUCTION}

For an even integer $k$, let us consider holomorphic Hecke forms of weight $k$ for the full modular group $\Gamma=P S L(2, \mathbb{Z})$. By the valence formula each such form has $k / 12+O(1)$ zeros in $\Gamma \backslash \mathcal{H}$. We are interested in the distribution of these zeros and write $\mathcal{Z}(f)$ for the zero set.

Rankin and Swinnerton-Dyer [16] have shown that all zeros of an Eisenstein series $E_{k}(z)$ are on the geodesic segment

$$
\delta_{3}=\{z \in \mathcal{H}:|z|=1,0 \leq \operatorname{Re} z \leq 1 / 2\} .
$$

Here we consider the rest of the Hecke eigenforms, that is the cusp forms. We write $H_{k}$ for the finite set of (Fourier-)normalized holomorphic Hecke cusp forms of weight $k$ for the full modular group $\Gamma$ and use the letter $f$ for a generic form in $H_{k}$. As a consequence of the holomorphic Quantum Unique Ergodicity conjecture, proved recently by Holowinsky and Soundararajan [7], we know by work of Rudnick [17] that $\mathcal{Z}(f)$ is equidistributed (with respect to the hyperbolic area) as $k \rightarrow \infty$.

In [3] Ghosh and Sarnak initiate the study of so-called real zeros of $f \in H_{k}$, that is zeros on three geodesic segments $\delta_{i}, i=1,2,3$. The segment $\delta_{3}$ is as above,

$$
\delta_{1}=\{z \in \mathcal{H}:|z|>1, \operatorname{Re}(z)=0\}, \quad \text { and } \quad \delta_{2}=\left\{z \in \mathcal{H}:|z|>1, \operatorname{Re}(z)=\frac{1}{2}\right\} .
$$

The zeros of $f$ are symmetric with respect to $\delta_{i}$ and the zeros in these segments are called real because $f(z)$ is real-valued on $\delta_{1}$ and $\delta_{2}$ while $z^{k / 2} f(z)$ is real-valued on $\delta_{3}$. For an illustrative figure on the distribution of the zeros in the fundamental domain, see [3, Figure 2].

Ghosh and Sarnak showed that the number of the real zeros grows quite rapidly with $k$ - indeed they proved that, for any $\varepsilon>0$ and large enough $k$,

$$
\left|\mathcal{Z}(f) \cap\left(\delta_{1} \cup \delta_{2}\right)\right| \gg_{\varepsilon} k^{1 / 4-1 / 80-\varepsilon}
$$

Date: June 6, 2014.

2000 Mathematics Subject Classification. 11N25, 11N36, $11 \mathrm{~F} 11$.

The author was supported by the Academy of Finland grant no. 137883. 
and that " $1 / 80$ " can be removed under the Riemann hypothesis. Here we will demonstrate that this removal can be done unconditionally.

Theorem 1.1. Let $k \rightarrow \infty$ and $f \in H_{k}$. Then

$$
\left|\mathcal{Z}(f) \cap\left(\delta_{1} \cup \delta_{2}\right)\right| \gg_{\varepsilon} k^{1 / 4-\varepsilon}
$$

for any $\varepsilon>0$.

In the previous theorem and in many statements below, an assumption of the type $x \rightarrow \infty$ means that the claim holds for all sufficiently large $x$.

Ghosh and Sarnak also showed that the number of zeros in each of the lines $\delta_{1}$ and $\delta_{2}$ goes to infinity with $k$ and the proof of this automatically gave a lower bound $\left|\mathcal{Z}(f) \cap \delta_{j}\right| \gg \log k$. Here we will prove a polynomial lower bound.

Theorem 1.2. Let $k \rightarrow \infty$ and $f \in H_{k}$. Then

$$
\left|\mathcal{Z}(f) \cap \delta_{1}\right| \gg k^{1 / 300}
$$

and

for any $\varepsilon>0$.

$$
\left|\mathcal{Z}(f) \cap \delta_{2}\right| \gg_{\varepsilon} k^{1 / 8-\varepsilon}
$$

The apparent weakness of the lower bound for the number of zeros in $\delta_{1}$ stems from weak knowledge about negative Hecke eigenvalues as can be seen from Theorem 5.5 below.

Furthermore Ghosh and Sarnak were led to a conjecture that there are $\asymp k^{1 / 2} \log k$ real zeros. This was backed up by both arithmetic considerations and a random model. Recalling that $\left|H_{k}\right| \asymp k$, the following theorem tells that the number of real zeros cannot often be of significantly smaller order.

Theorem 1.3. There exists an absolute constant $\eta>0$ such that, for any fixed $\varepsilon>0$ and for $k \rightarrow \infty$, there are at most $k^{1-\eta \varepsilon}$ forms $f \in H_{k}$ for which

$$
\left|\mathcal{Z}(f) \cap\left(\delta_{1} \cup \delta_{2}\right)\right| \leq k^{1 / 2-\varepsilon} .
$$

As will be explained in Remark 5.4, the reason why we have to contend with $k^{1 / 4-\varepsilon}$ rather than $k^{1 / 2-\varepsilon}$ when allowing no exceptional forms is that we cannot rule out the possibility that the normalized Fourier coefficients $\lambda_{f}(p)$ of $f$ have very small absolute values for all primes $p \leq k^{1 / 2}$.

We will describe the strategy of the proofs in detail in next section. To summarize, Ghosh and Sarnak [3] showed essentially that, for some values of $\operatorname{Im}(z)$, a single term dominates in the Fourier expansion

$$
f(z)=\sum_{n=1}^{\infty} \lambda_{f}(n) n^{(k-1) / 2} e(n z),
$$

where $e(x)=e^{2 \pi i x}$. Recall that the normalized Fourier coefficients $\lambda_{f}(n)$ are real and satisfy the Hecke relation

$$
\lambda_{f}(m) \lambda_{f}(n)=\sum_{d \mid(m, n)} \lambda_{f}\left(\frac{m n}{d^{2}}\right),
$$

so that in particular they are multiplicative and

$$
\lambda_{f}(p)^{2}=\lambda_{f}\left(p^{2}\right)+1 .
$$


Using these properties one quickly encounters a problem in multiplicative number theory: Show that many short intervals contain numbers with all prime factors in certain subsets of the primes. Section 4 is devoted to studying this problem, which, thought other way round, is a sieving problem.

In this paper we study only zeros $\alpha+i y$ with $y \gg k^{1 / 2} \log k$ and thereby zeros in $\delta_{1}$ and $\delta_{2}$. It would be interesting to gain understanding also about zeros with smaller $y$ and about zeros in $\delta_{3}$.

\section{Strategy}

Our approach follows that in the pioneering work of Ghosh and Sarnak [3]. A crucial ingredient is the following immediate consequence of [3, Theorem 3.1], which tells that, for some values of $y, f(\alpha+i y)$ is almost determined by a single Fourier coefficient $\lambda_{f}(l)$.

Proposition 2.1. There are positive constants $\beta_{1}, \beta_{2}$ and $\delta$ such that, for all integers $l \in\left(\beta_{1}, \beta_{2} \sqrt{k / \log k}\right)$ and all $f \in H_{k}$,

$$
\left(\frac{e}{l}\right)^{\frac{k-1}{2}} f\left(\alpha+i y_{l}\right)=\lambda_{f}(l) e(\alpha l)+O\left(k^{-\delta}\right)
$$

where $y_{l}=\frac{k-1}{4 \pi l}$.

Let $\alpha \in\{0,1 / 2\}$ and $\varepsilon^{\prime}>0$ be any fixed small constant, and let $k$ be large. Recalling that $f(\alpha+i y)$ is real for these $\alpha$, the proposition immediately implies that if we can find numbers $l_{1}, l_{2} \in\left(\beta_{1}, \beta_{2} \sqrt{k / \log k}\right)$ with

$$
\lambda_{f}\left(l_{1}\right) e\left(\alpha l_{1}\right)<-\varepsilon^{\prime}<\varepsilon^{\prime}<\lambda_{f}\left(l_{2}\right) e\left(\alpha l_{2}\right),
$$

then $f(z)$ has a zero $\alpha+i y$ with $y$ between $y_{l_{1}}$ and $y_{l_{2}}$.

This way we can get information about the zeros in

$$
\mathcal{F}_{Y}=\{z:-1 / 2<\operatorname{Re} z \leq 1 / 2, \operatorname{Im} z \geq Y\}
$$

with

$$
\beta_{1}^{\prime} \sqrt{k \log k} \leq Y \leq \beta_{2}^{\prime} k
$$

for certain positive constants $\beta_{1}^{\prime}$ and $\beta_{2}^{\prime}$.

If we are happy with zeros in either of the lines $\delta_{1}$ and $\delta_{2}$, it is enough to find $l_{1}$ and $l_{2}$ of different parity such that $\left|\lambda_{f}\left(l_{i}\right)\right| \geq \varepsilon^{\prime}$; for such $l_{1}$ and $l_{2}$ (interchanging them if necessary), (2) must hold with $\alpha=0$ or with $\alpha=1 / 2$. To find these $l_{1}$ and $l_{2}$ of different parity, write $\omega_{f}=2$, if $\left|\lambda_{f}(2)\right| \geq 1 / 2$ and $\omega_{f}=4$ otherwise. From (1) with $p=2$ we see that $\left|\lambda_{f}\left(\omega_{f}\right)\right| \geq 1 / 2$, and so it suffices to find odd $l_{1}^{\prime}$ and $l_{2}^{\prime}$ for which $\left|\lambda_{f}\left(l_{1}^{\prime}\right)\right|,\left|\lambda_{f}\left(l_{2}^{\prime}\right)\right| \geq 2 \varepsilon^{\prime}$ as we can take $l_{1}=\omega_{f} l_{1}^{\prime}$ and $l_{2}=l_{2}^{\prime}$.

Combining these observations and noticing that if we can find many consequtive pairs $l_{1}, l_{2}$, we get many zeros, we get the following proposition. There and later we say that a claim holds for proportion $\sigma$ of $y \in\left[C_{1} Y, C_{2} Y\right]$ if the measure of the set of those $y \in\left[C_{1} Y, C_{2} Y\right]$ for which the claim holds is at least $\sigma\left(C_{2}-C_{1}\right) Y$. Also, the notation $y \sim Y$ means $y \in[Y, 2 Y]$.

Proposition 2.2. Let $\varepsilon^{\prime}, \theta>0$ and $C \geq 1$ be fixed and $k \rightarrow \infty$. Let $Y$ be as in (4) and $X=k /(20 Y)$. 
(i) Let $j \in\{1,2\}$. Assume that for a positive proportion of $x \sim X$ there exist two distinct numbers $l_{1}, l_{2} \in\left[x, x+C X^{\theta}\right]$ for which

$$
(-1)^{(j-1) l_{1}} \lambda_{f}\left(l_{1}\right)<-\varepsilon^{\prime}<\varepsilon^{\prime}<(-1)^{(j-1) l_{2}} \lambda_{f}\left(l_{2}\right) .
$$

Then

$$
\left|\mathcal{Z}_{f} \cap \delta_{j} \cap \mathcal{F}_{Y}\right| \gg X^{1-\theta}
$$

(ii) Assume that for a positive proportion of $x \sim X$ there exist odd $l_{1} \in\left[x, x+C X^{\theta}\right]$ and $l_{2} \in\left[x / \omega_{f},\left(x+C X^{\theta}\right) / \omega_{f}\right]$ for which $\left|\lambda_{f}\left(l_{1}\right)\right|,\left|\lambda_{f}\left(l_{2}\right)\right| \geq \varepsilon^{\prime}$. Then

$$
\left|\mathcal{Z}_{f} \cap\left(\delta_{1} \cup \delta_{2}\right) \cap \mathcal{F}_{Y}\right| \gg X^{1-\theta} .
$$

Intervals around $x$ and $x / \omega_{f}$ are completely analogous and so it is enough to consider only the intervals $\left[x, x+C X^{\theta}\right]$. But to guarantee that for positive proportion of $x \sim X$ one finds wanted numbers both in $\left[x, x+C X^{\theta}\right]$ and in $\left[x / \omega_{f},\left(x+C X^{\theta}\right) / \omega_{f}\right]$, we need to show that a proportion strictly larger than half of both collections of intervals are "good".

Since we are dealing with rather small $l_{i}\left(l_{i} \ll \sqrt{k / \log k}\right)$, it is difficult to say much about the Fourier coefficients, although for almost all forms, a large sieve inequality works well even for small $l_{i}$. Fortunately (1) comes to our rescue: it implies a much-exploited property (first used by Iwaniec and Sarnak [8]) that $\left|\lambda_{f}(p)\right|$ and $\left|\lambda_{f}\left(p^{2}\right)\right|$ cannot be small simultaneously. Let $\eta>0$ and write, for $j=1,2$,

(5) $\mathcal{P}_{j}=\left\{p \in\left[X^{\eta}, X\right]:\left|\lambda_{f}\left(p^{j}\right)\right| \geq 1 / 5\right\} \quad$ and $\quad \mathcal{N}_{j}=\left\{n \in \mathbb{N}: p \mid n \Longrightarrow p \in \mathcal{P}_{j}\right\}$.

Then

$$
\mathcal{P}_{1} \cup \mathcal{P}_{2}=\mathbb{P} \cap\left[X^{\eta}, X\right]
$$

by (1) and if $n_{j} \in \mathcal{N}_{j} \cap[1,3 X]$ is square-free, then by multiplicativity $\left|\lambda_{f}\left(n_{j}^{j}\right)\right| \geq$ $(1 / 5)^{2 / \eta}$ (since $n$ has at most $2 / \eta$ prime divisors). Notice that $\mathcal{N}_{j} \cap[1,3 X]$ contains at most $3 X^{1-\eta}$ non-square-free numbers and so $\mathcal{N}_{j} \cap\left[x, x+C X^{\theta}\right]$ contains more than $X^{\theta} /(\log X)^{10}$ non-square-free numbers for $o(X)$ of $x \sim X$.

Combining these observations we have the following proposition, where and later we write, for any set $\mathcal{C} \subseteq \mathbb{R}$ and real numbers $x \leq y, \mathcal{C}(x, y)=\mathcal{C} \cap[x, y]$.

Proposition 2.3. Let $\delta, \theta \in(0,1], C \geq 1, k \rightarrow \infty$ and $f \in H_{k}$. Let $\mathcal{P}_{j}$ and $\mathcal{N}_{j}$ be as in (5), $Y$ as in (4) and $X=k /(20 Y)$.

(i) Assume that, for $\nu=1$ and for $\nu=\omega_{f}$, one has, for proportion at least $1 / 2+\delta$ of $x \sim X$

$$
\mathcal{N}_{1}\left(x / \nu,\left(x+C X^{\theta}\right) / \nu\right) \gg X^{\theta} /(\log X)^{5}
$$

Then

$$
\left|\mathcal{Z}_{f} \cap\left(\delta_{1} \cup \delta_{2}\right) \cap \mathcal{F}_{Y}\right| \gg X^{1-\theta} .
$$

(ii) Assume that, for $\nu=1$ and for $\nu=\omega_{f}$, one has, for proportion at least $1 / 2+\delta$ of $x \sim X^{1 / 2}$,

$$
\mathcal{N}_{2}\left(x / \nu^{1 / 2},\left(x+C X^{\theta}\right) / \nu^{1 / 2}\right) \gg X^{\theta} /(\log X)^{5} .
$$

Then

$$
\left|\mathcal{Z}_{f} \cap\left(\delta_{1} \cup \delta_{2}\right) \cap \mathcal{F}_{Y}\right| \gg X^{1 / 2-\theta}
$$


We will show that when $\varepsilon>0$ and $\eta$ is small enough (depending on $\varepsilon$ ), either (7) holds for almost all $x \sim X^{1 / 2}$ with $\theta=\varepsilon$, or

$$
\mathcal{N}_{1}\left(x / \nu^{1 / 2},\left(x+C X^{\varepsilon}\right) / \nu^{1 / 2}\right) \gg X^{\varepsilon} /(\log X)
$$

for certain proportion $\beta$ of $x \in\left[C_{1} X^{1 / 2}, C_{2} X^{1 / 2}\right]$ for any constants $C_{2}>C_{1}>0$. In the latter case we can multiply numbers from these sets to find that (6) holds with $\theta=1 / 2+\varepsilon$ for proportion $\beta-o(1)$ of $x \sim X$. Actually if $\beta>1 / 2$, one could use the pigeon-hole principle to show that (6) holds for all $x \sim X$. A similar idea was introduced by Croot [1] into the study of smooth numbers in short intervals.

Unfortunately we can only show (8) for proportion $1 / 2-o(1)$ of $x \in\left[C_{1} X^{1 / 2}, C_{2} X^{1 / 2}\right]$. To recover the proportion $1 / 2+\delta$ of $x \sim X$ needed in Proposition 2.3, we will use a variant of Freiman's $3 k-3$ theorem from additive combinatorics.

We will prove the necessary lemmas for studying $\mathcal{N}_{i}\left(x, x+C X^{\theta}\right)$ in Section 4 utilizing tools used in considerations of primes in almost all short intervals as well as sieve methods and respective results on long intervals. Some auxiliary results are introduced in Section 3 and the main theorems will be proved in Section 5.

The difference to the approach of Ghosh and Sarnak [3] is that they only looked for primes in short intervals rather than products of primes, that is they considered $\mathcal{P}_{1} \cup \mathcal{P}_{2}=\mathbb{P}$ rather than $\mathcal{N}_{1} \cup \mathcal{N}_{2}$ in short intervals. This left room for improvement since almost all intervals $\left[x, x+X^{\varepsilon}\right]$ are unconditionally known to contain primes only for $\varepsilon>1 / 20$ (see [9]), whereas we will be able to say something for any $\varepsilon>0$.

\section{Auxiliary Results}

We start by stating the Rosser-Iwaniec upper bound $\beta$-sieve (see [2, Chapter 11, in particular Theorem 11.12]) in a form which is convenient to us. The parameters $\kappa$ and $D$ below are respectively called dimension and level of the sieve.

Lemma 3.1. Let $D \geq 1$ and $\kappa>0$. There exists a sequence $\left(\lambda_{d}^{+}\right)_{d \in \mathbb{N}}$, a real number $\beta_{\kappa}$ and a function $F_{\kappa}: \mathbb{R}_{+} \rightarrow \mathbb{R}_{\geq 0}$ (depending only on $\kappa$ ) such that the following hold.

(1) $\lambda_{1}^{+}=1, \quad \lambda_{d}^{+}=0$ for $d>D$ and $\left|\lambda_{d}^{+}\right| \leq 1$ for all $d$;

(2) For all $n>1$,

$$
\sum_{d \mid n} \lambda_{d}^{+} \geq 0
$$

(3) Let $z=D^{1 / s}$ with $s>0$. For all multiplicative functions $g(d)$ satisfying $0 \leq$ $g(p)<1$ for all $p \in \mathbb{P}$, and

$$
\prod_{w_{1} \leq p<w_{2}}(1-g(p))^{-1} \leq\left(1+\frac{L}{\log w_{1}}\right)\left(\frac{\log w_{2}}{\log w_{1}}\right)^{\kappa}
$$

for all $w_{2}>w_{1} \geq 2$ and some constant $L \geq 1$, one has

$$
\sum_{d \mid \prod_{p<z} p} \lambda_{d}^{+} g(d) \leq\left(F_{\kappa}(s)+O_{\kappa, L}\left((\log D)^{-1 / 6}\right)\right) \prod_{p<z}(1-g(p)) \quad \text { for } s>\beta_{\kappa}-1 .
$$

(4) $\beta_{\kappa} \in[1,2]$ for $\kappa \in[1 / 2,1]$;

(5) $F_{\kappa}(s)=1+O_{\kappa}\left(e^{-s}\right), F_{1 / 2}(1)=2 \sqrt{e^{\gamma} / \pi}$ and $F_{\kappa}(s)$ is continuous with respect to $\kappa$ and $s$. 
In the proof of Theorem 1.3 we will use a large sieve inequality for the Fourier coefficients $\lambda_{f}(n)$. The version we use is the following special case $N=j=1$ of a more general theorem [12, Theorem 1] due to Lau and $\mathrm{Wu}$.

Lemma 3.2. Let $\nu \geq 1$ be a fixed integer and let $\left(b_{p}\right)_{p \in \mathbb{P}}$ be such that $\left|b_{p}\right| \leq B$ for all primes $p$. Then

$$
\sum_{f \in H_{k}}\left|\sum_{P<p \leq Q} b_{p} \frac{\lambda_{f}\left(p^{\nu}\right)}{p}\right|^{2} \ll_{\nu} k \frac{B^{2}}{P \log P}+k^{10 / 11} \frac{B^{2} Q^{\nu / 5}}{(\log P)^{2}}
$$

uniformly for

$$
B>0, \quad 2 \mid k, \quad 2 \leq P<Q \leq 2 P .
$$

To relate short intervals to long intervals, we will use so-called type II information used in studying primes in almost all short intervals. Here and later, when we say that a statement holds for almost all $x \in[X, 2 X]$, we mean that, for any $A$, the measure of the exceptional set is $O_{A}\left(X /(\log X)^{A}\right)$. We will also write $\tau(n)$ for the divisor function.

Lemma 3.3. Let $\varepsilon>\eta>0, A \geq 1, y=x(2 X)^{\varepsilon-1}$ and $y_{1}=x \exp \left(-3(\log X)^{1 / 3}\right)$. Let

$$
M(s)=\sum_{m \sim M} a_{m} m^{-s} \quad \text { and } \quad R(s)=\sum_{r \sim R} c_{r} r^{-s}
$$

be Dirichlet polynomials with $a_{m} \ll \tau(m)^{C}$ for some absolute constant $C$ and with $c_{r}$ a characteristic function of an interval or of primes in an interval. Suppose that $M R=X$ and $R=X^{\beta}$. If $\beta \in[\eta, \varepsilon]$, then

$$
\sum_{x \leq m r \leq x+y} a_{m} c_{r}=\frac{y}{y_{1}} \sum_{x \leq m r \leq x+y_{1}} a_{m} c_{r}+O_{\varepsilon, \eta, A, C}\left(y(\log X)^{-A}\right)
$$

for almost all $x \in[X, 2 X]$.

Proof. See [5, Lemma 9.3 and the discussion below it]. The condition $\theta>1 / 25$ present in [5, Chapter 9] is not needed for this lemma. Also the polynomial $R(s)$ satisfies the needed hypothesis [5, (7.2.3)]: When $R(s)$ is a relative of the characteristic function of primes, this is for instance [5, Lemma 1.5] and the other case is easier.

Notice that in the previous lemma $M$ and $R$ must be in a very narrow range. As often, this problem will be overcome by considering only products $m r$, where $m$ belongs to a well-behaving subset of $\mathcal{N}$ and sieving is applied only to $r$. There are more sophisticated versions of Lemma 3.3 with somewhat wider ranges for $M$ and $R$ (see for instance [5, Lemma 9.4]), but we do not need them here.

As mentioned in the previous section, in case of $\mathcal{N}_{1}$, when we pass from very short intervals $\left[x, x+X^{\varepsilon}\right]$ with $x \sim X^{1 / 2}$ to longer intervals $\left[x, x+C X^{1 / 2+\varepsilon}\right]$ with $x \sim X$, we will sometimes need to be able to increase the proportion of "good" intervals a bit. This will be done with help of properties of sets with small doubling, that is sets $A$ for which $|A+A|$ is small. Here, for sets $A, B \subseteq \mathbb{Z}$, we use the notation

$$
A+B=\{a+b: a \in A, b \in B\} .
$$

Freiman's $3 k-3$-theorem (see [19, Theorem 5.11]) tells us that any finite set $A \subset \mathbb{Z}$ with $|A+A|<3|A|-3$ is contained in a relatively short arithmetic progression, 
but here we will need information also about the number of representations

$$
r_{A+A}(c)=\{(a, b) \in A \times A: a+b=c\} .
$$

Lemma 3.4. Let $\eta \in(0,1 / 100)$ and $n \geq 1$. If $A \subseteq\{1, \ldots, n\}$ is such that $|A| \geq 3$ and

$$
\left|\left\{c \in A+A: r_{A+A}(c) \geq \eta^{2} \frac{|A|^{2}}{|A+A|}\right\}\right| \leq\left(\frac{5}{2}-3 \eta\right)|A|-2,
$$

then there exists an arithmetic progression $P$ such that

$$
|A \cap P| \geq(1-\eta)|A| \quad \text { and } \quad|P| \leq \frac{3}{2}|A| .
$$

The proof is based on the following lemma, where we write, for $E \subseteq A \times B$,

$$
A \stackrel{E}{+} B=\{a+b:(a, b) \in E\} .
$$

Lemma 3.5. Let $A \subseteq\{0, \ldots, \ell\}$ be such that $0, \ell \in A,|A| \geq 3$ and $\operatorname{gcd}(A)=1$. Suppose that $E \subseteq A \times A$ and $K \in \mathbb{N}$ are such that

(i) for every $a \in A$, there correspond at most $K$ elements $b \in A$ such that $(a, b) \notin$ $E$ and at most $K$ elements $b^{\prime} \in A$ such that $\left(b^{\prime}, a\right) \notin E$;

(ii) If $r_{A+A}(c) \geq K$, then $c \in A \stackrel{E}{+} A$.

Then

$$
|A \stackrel{E}{+} A| \geq \begin{cases}|A|+\ell-2 K & \text { if } \ell \leq 2|A|-2 K-3 \\ \frac{3+\sqrt{5}}{2}|A|-6 K-2 & \text { if } \ell \geq 2|A|-2 K-2 .\end{cases}
$$

Proof. This follows immediately from Lev [13, Theorem 1] by taking $s=K=K$ and

$$
\mathcal{R}=|\{(a, b) \in A \times A: a+b \notin \stackrel{E}{E} A\}| \subseteq(A \times A) \backslash E
$$

there (notice that $\stackrel{\mathcal{R}}{+} A$ in [13] has complementary meaning to $A \stackrel{E}{+} A$ here).

Proof of Lemma 3.4. For

$$
E=\left\{(a, b) \in A \times A: r_{A+A}(a+b) \geq \eta^{2} \frac{|A|^{2}}{|A+A|}\right\},
$$

one has $|A|^{2} \leq|E|+|A+A| \cdot \eta^{2} \frac{|A|^{2}}{|A+A|}$, so that $|E| \geq\left(1-\eta^{2}\right)|A|^{2}$. Take

$$
\begin{aligned}
A^{\prime} & =\{a \in A:(a, b) \notin E \text { for at most } \eta|A| \text { of } b \in A\} \\
& =\{a \in A:(a, b) \in E \text { for at least }(1-\eta)|A| \text { of } b \in A\} .
\end{aligned}
$$

Now

$$
\left(1-\eta^{2}\right)|A|^{2} \leq|E| \leq\left|A^{\prime}\right||A|+\left(|A|-\left|A^{\prime}\right|\right)(1-\eta)|A| \Longrightarrow\left|A^{\prime}\right| \geq(1-\eta)|A| .
$$

By definitions of $E$ and $A^{\prime}$, the sets $A^{\prime}$ and $E^{\prime}=E \cap\left(A^{\prime} \times A^{\prime}\right)$ satisfy assumptions (i) and (ii) of Lemma 3.5 with $K=\lfloor\eta|A|\rfloor$. Shifting $A^{\prime}$ (and $E^{\prime}$ ) to the left by $\min A^{\prime}=x_{0}$, say, and then dividing by the largest common divisor $d$, say, we obtain a set $A^{\prime \prime} \subseteq\{0, \ldots, \ell\}$ such that $0, \ell \in A^{\prime \prime}, \operatorname{gcd}\left(A^{\prime \prime}\right)=1$, and a set $E^{\prime \prime}$ with similar properties as $E^{\prime}$. 
Applying Lemma 3.5 with $K=\lfloor\eta|A|\rfloor$, we get that either $\ell \leq 3|A| / 2-1$ or

$$
\begin{aligned}
& \left|\left\{c \in A+A: r_{A+A}(c) \geq \eta^{2} \frac{|A|^{2}}{|A+A|}\right\}\right| \geq\left|A^{\prime} \stackrel{E^{\prime}}{+} A^{\prime}\right|=\left|A^{\prime \prime} \stackrel{E^{\prime \prime}}{+} A^{\prime \prime}\right| \\
& \geq \min \left\{\left|A^{\prime}\right|+\frac{3}{2}|A|-1-2 \eta|A|, \frac{3+\sqrt{5}}{2}\left|A^{\prime}\right|-6 \eta|A|-2\right\}>\left(\frac{5}{2}-3 \eta\right)|A|-2
\end{aligned}
$$

since $\eta<1 / 100$. Hence, by assumption, we must have $\ell \leq 3|A| / 2-1$, in which case one can take $P=\left\{x_{0}+j d: j=0, \ldots, \ell\right\} \supseteq A^{\prime}$.

\section{Sieving SHORT INTERVALS}

Let $\mathcal{P}$ be a subset of the primes and consider the set

$$
\mathcal{N}=\{n \in \mathbb{N}: p \mid n \Longrightarrow p \in \mathcal{P}\}=\left\{n \in \mathbb{N}: p \mid n \Longrightarrow p \notin \mathcal{P}^{c}\right\} .
$$

We are interested in the short interval distribution of $\mathcal{N}$. This question was studied by Harman and the author in [6], where we used a $1-\tau$-dimensional sieve to prove the following result.

Lemma 4.1. Let $\tau \in(1 / 2,1]$ and assume that

$$
\sum_{p \in \mathcal{P}(2, x)} \frac{\log p}{p}=\tau \log x+O(1)
$$

for all $x \geq 2$. Then there exists a positive constant $\delta=\delta(\tau)$ such that

$$
\left|\mathcal{N}\left(x, x+x^{1-\delta}\right)\right| \gg \frac{x^{1-\delta}}{(\log x)^{1-\tau}}
$$

for all large enough $x$.

Unfortunately this is nowhere near what we need - we want to find numbers in shorter intervals and with a more wildly behaving set $\mathcal{P}$. In Subsection 4.2 we will show that if we have more than half of the primes just in one interval $\left[x^{\beta_{1}}, x^{\beta_{2}}\right]$, then, for any $\varepsilon>0$, we get a lower bound of the correct order of magnitude for $\left|\mathcal{N}\left(x, x+X^{\varepsilon}\right)\right|$ for almost all $x \sim X$ and for $\left|\mathcal{N}\left(x, x+x^{1 / 2+\varepsilon}\right)\right|$ for all large enough $x$. In Subsection 4.3 we will show that even if (10) holds for some $\tau \leq 1 / 2$, we can find a lower bound of the correct order for $\left|\mathcal{N}\left(x, x+X^{\varepsilon}\right)\right|$ for a certain proportion of $x \sim X$.

We will prove statements that are stronger than we need - in particular we would be happy with finding fewer square-free numbers in each interval, and this could be done for example with a weaker auxiliary result than Lemma 4.3 below. However here we aim to prove lower bounds of the right order of magnitude under weak assumptions which might be of benefit in further applications.

4.1. Long intervals. At the end we want to prove that even short intervals contain numbers in $\mathcal{N}$. However, when we do that we need to understand long intervals well and we start by quoting results concerning them.

Our first result is an asymptotic formula for the mean value of any well-behaving multiplicative function. The argument goes back to Wirsing [20] but we quote a more general statement which is [2, Theorem A.5]. 
Lemma 4.2. Let $k>-1 / 2$. Suppose that $g$ is a multiplicative function which satisfies

$$
\sum_{p \leq x} g(p) \log p=k \log x+O(1)
$$

for all $x \geq 2$. Assume further that

$$
\prod_{w \leq p<z}(1+|g(p)|) \ll\left(\frac{\log z}{\log w}\right)^{|k|} \quad \text { for all } z>w \geq 2
$$

and

$$
\sum_{p \in \mathbb{P}} g(p)^{2} \log p<\infty
$$

Then

$$
\sum_{n \leq x} g(n)|\mu(n)|=\frac{(\log x)^{k}}{\Gamma(k+1)} \prod_{p \in \mathbb{P}}\left(1-\frac{1}{p}\right)^{k}(1+g(p))+O\left((\log x)^{|k|-1}\right) .
$$

The following lemma which is a direct consequence of $[4$, Theorem 1 combined with Remark 1.4] shows that, under a mild assumption, $|\mathcal{N}(x,(1+\delta) x)|$ is of the expected order of magnitude. This will allow us to generate very regular subsets $\mathcal{N}^{\prime} \subset \mathcal{N}$.

Lemma 4.3. There exists an absolute constant $\lambda>1$ such that if $\delta, \eta>0$ and $x \rightarrow \infty$, then the following holds. If $\mathcal{P}$ is a subset of the primes for which

$$
\sum_{p \in \mathcal{P}\left(x^{\eta}, x\right)} \frac{1}{p} \geq \lambda
$$

then

$$
|\mathcal{N}(x,(1+\delta) x)| \gg_{\eta} \delta x \prod_{p \in \mathcal{P}^{c}(2, x)}\left(1-\frac{1}{p}\right) .
$$

4.2. When $\mathcal{P}$ contains more than half of the primes somewhere. Using a very simple sieve idea together with Lemmas 3.3 and 4.3 we can prove the following result.

Lemma 4.4. Let $\lambda$ be as in Lemma 4.3, let $\delta, \varepsilon, \eta>0$ with $\eta<\min \{\varepsilon, 1-2 \varepsilon\}$, and let $X \rightarrow \infty$. Assume that there exists $w, z \in\left[X^{\eta}, X^{\varepsilon}\right]$ with $z>(1+\eta)$ w such that

$$
\sum_{p \in \mathcal{P}(w, z)} \frac{1}{p} \geq(1 / 2+\delta) \sum_{p \in \mathbb{P}(w, z)} \frac{1}{p}
$$

Assume further that

$$
\sum_{p \in \mathcal{P}\left(X^{\eta}, X^{1-2 \varepsilon}\right)} \frac{1}{p} \geq \lambda
$$

Then

$$
\left|\mathcal{N}\left(x, x+X^{\varepsilon}\right)\right| \gg_{\varepsilon, \delta, \eta} X^{\varepsilon}\left(\frac{\log \frac{z}{w}}{\log X}\right)^{2} \prod_{p \in \mathcal{P}^{c}(2, X)}\left(1-\frac{1}{p}\right)
$$

for almost all $x \in[X, 2 X]$. 
Proof. Let $\eta^{\prime}$ be a small positive constant (small compared to $\delta$ ). By restricting to a subinterval it is enough to prove the statement when $\frac{\log z}{\log w}<1+\eta^{\prime}$.

Let $\mathcal{N}^{\prime} \subset \mathcal{N}\left(X / z^{2}, 2 X / w^{2}\right)$ and $c_{\eta}>0$ be such that

$$
\sum_{n \in \mathcal{N}^{\prime}(x / z, x / w)} \frac{1}{n} \in\left[1-\eta^{\prime}, 1+\eta^{\prime}\right] \cdot c_{\eta} \log \frac{z}{w} \prod_{p \in \mathcal{P}^{c}(2, X)}\left(1-\frac{1}{p}\right)
$$

for all $x \in[X / z, 2 X / w]$. Such set $\mathcal{N}^{\prime}$ can be found by dividing $\left[X / z^{2}, 2 X / w^{2}\right]$ into disjoint subintervals $\left[x,\left(1+\eta^{\prime 2}\right) x\right]$ and using Lemma 4.3 to find

$$
c_{\eta} \eta^{2} x \prod_{p \in \mathcal{P}^{c}(2, X)}\left(1-\frac{1}{p}\right)+O(1)
$$

members of $\mathcal{N}$ from each of these (here $c_{\eta}$ is the implied constant in Lemma 4.3).

Write

$$
\mathcal{A}=\left\{p_{1} p_{2} n: p_{i} \in \mathcal{P}(w, z), n \in \mathcal{N}^{\prime}\right\},
$$

where elements are counted with multiplicity (which is at most $2 / \eta^{2} \ll 1$ for $n \in$ $\mathcal{A}(1,3 X))$. Now, for any $x_{2} \geq x_{1} \geq 1$,

$$
\begin{aligned}
\left|\mathcal{A}\left(x_{1}, x_{2}\right)\right| \geq & \left|\left\{p_{1} p_{2} n \in\left[x_{1}, x_{2}\right]: p_{1} \in \mathcal{P}(w, z), p_{2} \in \mathbb{P}(w, z), n \in \mathcal{N}^{\prime}\right\}\right| \\
& -\left|\left\{p_{1} p_{2} n \in\left[x_{1}, x_{2}\right]: p_{1} \in \mathbb{P}(w, z), p_{2} \in \mathcal{P}^{c}(w, z), n \in \mathcal{N}^{\prime}\right\}\right| \\
= & \left|\mathcal{A}_{1}\left(x_{1}, x_{2}\right)\right|-\left|\mathcal{A}_{2}\left(x_{1}, x_{2}\right)\right|,
\end{aligned}
$$

say.

The point is that in each $\mathcal{A}_{i}$ there is a variable ranging over all primes in an interval, so that we can apply Lemma 3.3. Besides, since the relative density of $\mathcal{P}(w, z)$ is strictly more than half, one expects that $\mathcal{A}_{1}$ is larger than $\mathcal{A}_{2}$, so that we do not lose too much in (12). Our next task is to confirm this line of thought.

Let $y=x(2 X)^{\varepsilon-1}$ and $y_{1}=x \exp \left(-3(\log X)^{1 / 3}\right)$. By Lemma 3.3 with $r=p_{2}$, we have

$$
\left|\mathcal{A}_{1}(x, x+y)\right|=\sum_{\substack{x \leq m p_{2} \leq x+y \\ p_{2} \in \mathbb{P}(w, z)}}\left(\sum_{\substack{m=p_{1} n \\ p_{1} \in \mathcal{P}(w, z), n \in \mathcal{N}^{\prime}}} 1\right)=\frac{y}{y_{1}}\left|\mathcal{A}_{1}\left(x, x+y_{1}\right)\right|+O\left(y(\log X)^{-A}\right)
$$

for almost all $x \in[X, 2 X]$.

Furthermore

$$
\begin{aligned}
\left|\mathcal{A}_{1}\left(x, x+y_{1}\right)\right| & =\sum_{p_{1} \in \mathcal{P}(w, z)} \sum_{n \in \mathcal{N}^{\prime}} \sum_{\substack{p_{2} \in \mathbb{P}(w, z) \\
\frac{x}{n p_{1}} \leq p_{2} \leq \frac{x+y_{1}}{n p_{1}}}} 1 \\
& \geq(1-o(1)) \frac{y_{1}}{\log z} \sum_{p_{1} \in \mathcal{P}(w, z)} \frac{1}{p_{1}} \sum_{\substack{n \in \mathcal{N}^{\prime}\left(\frac{x}{z p_{1}}, \frac{x}{w p_{1}}\right) \\
n}} \frac{1}{n} \\
& \geq(1-o(1)) \frac{y_{1}}{\log z}\left(\frac{1}{2}+\delta\right)\left(\sum_{w \leq p \leq z} \frac{1}{p}\right) c_{\eta}\left(1-\eta^{\prime}\right) \log \frac{z}{w} \prod_{p \in \mathcal{P}^{c}(2, X)}\left(1-\frac{1}{p}\right) .
\end{aligned}
$$


Similarly $\left|\mathcal{A}_{2}(x, x+y)\right|=\frac{y}{y_{1}}\left|\mathcal{A}_{2}\left(x, x+y_{1}\right)\right|+O\left(y(\log X)^{-A}\right)$ for almost all $x \sim X$ and

$$
\left|\mathcal{A}_{2}\left(x, x+y_{1}\right)\right| \leq(1+o(1)) \frac{y_{1}}{\log w}\left(\frac{1}{2}-\delta\right)\left(\sum_{w \leq p \leq z} \frac{1}{p}\right) c_{\eta}\left(1+\eta^{\prime}\right) \log \frac{z}{w} \prod_{p \in \mathcal{P}^{c}(2, X)}\left(1-\frac{1}{p}\right) .
$$

Since $\frac{\log z}{\log w}<1+\eta^{\prime}$ and since $\eta^{\prime}$ is small compared to $\delta$, we get from (12)-(15) that

$$
|\mathcal{A}(x, x+y)| \geq\left|\mathcal{A}_{1}(x, x+y)\right|-\left|\mathcal{A}_{2}(x, x+y)\right| \gg y \frac{\log \frac{z}{w}}{\log x}\left(\sum_{w \leq p \leq z} \frac{1}{p}\right) \prod_{p \in \mathcal{P}^{c}(2, X)}\left(1-\frac{1}{p}\right)
$$

for almost all $x \in[X, 2 X]$. This implies the claim since $\left|\mathcal{N}\left(x, x+X^{\varepsilon}\right)\right| \gg \mid \mathcal{A}(x, x+$ $y) \mid$ for all $x \sim X$ and

$$
\sum_{w \leq p \leq z} \frac{1}{p} \gg \frac{\log \frac{z}{w}}{\log w}
$$

when $\log z \ll \log w$.

The assumption (11) in the previous lemma could be relaxed to

$$
\sum_{\substack{p \in \mathcal{P}(w, z) \\ p \equiv a(\bmod q)}} \frac{1}{p} \geq(1 / 2+\delta) \sum_{\substack{p \in \mathbb{P}(w, z) \\ p \equiv a(\bmod q)}} \frac{1}{p}
$$

for some $w$ and $z$ as in the lemma and some bounded co-prime $a$ and $q$. The proof could also be modified to give the following improvement of Lemma 4.1.

Lemma 4.5. Let $\lambda$ be as in Lemma 4.3 and let $\delta, \varepsilon, \eta>0$ with $\eta<\min \{\varepsilon / 2,1 / 2-\varepsilon\}$ and let $x \rightarrow \infty$. Assume that there exists $w, z \in\left[x^{\eta}, x^{\varepsilon}\right]$ with $z>(1+\eta) w$ such that

$$
\sum_{p \in \mathcal{P}(w, z)} \frac{1}{p} \geq(1 / 2+\delta) \sum_{p \in \mathbb{P}(w, z)} \frac{1}{p}
$$

Assume further that

$$
\sum_{p \in \mathcal{P}\left(x^{\eta}, x^{1 / 2-\varepsilon}\right)} \frac{1}{p} \geq \lambda
$$

Then

$$
\left|\mathcal{N}\left(x, x+x^{1 / 2+\varepsilon}\right)\right| \gg_{\varepsilon, \delta, \eta} x^{1 / 2+\varepsilon}\left(\frac{\log \frac{z}{w}}{\log x}\right)^{2} \prod_{p \in \mathcal{P}^{c}(2, x)}\left(1-\frac{1}{p}\right) .
$$

Proof. This time one could take

$$
\mathcal{A}=\left\{p_{1} p_{2} n_{1} n_{2}: p_{i} \in \mathcal{P}(w, z), n_{i} \geq x^{1 / 2-\varepsilon}, n_{i} \in \mathcal{N}_{i}^{\prime}\right\},
$$

where $\mathcal{N}_{i}^{\prime} \subset \mathcal{N}$ are very regular large sets with $\mathcal{N}_{2}^{\prime}$ consisting only of numbers with all prime factors greater than $x^{\eta}$ (to avoid counting same numbers too many times) and use type II information for primes in all intervals problem (see for instance [5, Section 7.2]). 
4.3. When $\mathcal{P}$ is regular. Next we prove a lemma which we will use when we do not have strictly more than half of the primes in any interval. As we will be considering a decomposition $\mathbb{P}=\mathcal{P}_{1} \cup \mathcal{P}_{2}$ at the end, this will mean that both sets contain approximatively half of the primes all the time and we are able to use a classical sieve method.

In the proof of the following lemma we will be using constants $\eta_{5}>\eta_{4}>\eta_{3}>$ $\eta_{2}>\eta_{1}$ (with $\eta_{5}$ given), each significantly smaller than the previous one. When we write $o(1)$ in the proof, we mean that the quantity tends to 0 when these constants (except $\eta_{5}$ ) tend to 0 and their ratios tend to infinity.

Presence of so many parameters might look confusing at first, so we summarize approximate meaning of each here: We will be looking for numbers $m n$ with $n$ from a well-behaving subset of $\mathcal{N}$ and $m \in\left[X^{\eta_{4}}, X^{\eta_{5}}\right]$ with all prime factors at least $X^{\eta_{2}}$. We will use a linear sieve $\left(\kappa=1\right.$ ) with $z=X^{\eta_{2}}$ and $D=X^{\eta_{3}}$ (so that $s=\eta_{3} / \eta_{2}$ is large) to detect this condition. The smallest constant $\eta_{1}$ is used like $\eta^{\prime}$ in the previous subsection; it is a tiny parameter whose presence affects everything very little.

Lemma 4.6. Let $\varepsilon, \varepsilon^{\prime}, \eta_{5}>0, \kappa \in(0,1)$ and $X \rightarrow \infty$. Let $F_{\kappa}(s)$ be as in Lemma 3.1. There exists a small positive constant $\eta_{2}=\eta_{2}\left(\varepsilon, \varepsilon^{\prime}, \eta_{5}, \kappa\right)$ such that if

$$
\sum_{p \in \mathcal{P}^{c}(w, z)} \frac{1}{p} \leq \kappa \sum_{p \in \mathbb{P}(w, z)} \frac{1}{p}
$$

for all $w, z \in\left[X^{\eta_{2}}, X^{\eta_{5}}\right]$ with $z>\left(1+\eta_{2}\right) w$, then

$$
\mathcal{N}\left(\left[x, x+X^{\varepsilon}\right]\right) \gg_{\varepsilon, \varepsilon^{\prime}, \kappa, \eta_{5}} X^{\varepsilon} \prod_{p \in \mathcal{P}^{c}(2, X)}\left(1-\frac{1}{p}\right)
$$

for at least proportion

$$
\frac{e^{\gamma \kappa}}{F_{\kappa}(1) \Gamma(1-\kappa)}-\varepsilon^{\prime}
$$

of $x \in[X, 2 X]$.

Proof. As said before the lemma, we take very small constants $\eta_{1}<\eta_{2}<\eta_{3}<\eta_{4}<$ $\eta_{5}$ such that $\eta_{i+1} / \eta_{i}$ is large. We can clearly assume that $\eta_{5}+2 \eta_{1}<\varepsilon$ and that $\varepsilon^{\prime}$ is small. Further, without essentially affecting the final claim, we can enlarge the set $\mathcal{P}^{c}\left(X^{\eta_{2}}, X^{\eta_{5}}\right)$ so that

$$
\sum_{p \in \mathcal{P}^{c}(w, z)} \frac{1}{p}=\kappa \sum_{p \in \mathbb{P}(w, z)} \frac{1}{p}+O\left(\frac{1}{\log w}\right)
$$

for all $X^{\eta_{2}} \leq w<z \leq X^{\eta_{5}}$, and consequently by partial summation

$$
\sum_{p \in \mathcal{P}^{c}(w, z)} \frac{\log p}{p}=\kappa \log \frac{z}{w}+O(1)
$$

for all $X^{\eta_{2}} \leq w<z \leq X^{\eta_{5}}$

This time we take $\mathcal{N}^{\prime} \subset \mathcal{N}$ and $c_{\eta_{2}}>0$ such that

$$
\sum_{n \in \mathcal{N}^{\prime}\left(x,\left(1+\eta_{1}\right) x\right)} 1 \in\left[1-\eta_{1}, 1+\eta_{1}\right] c_{\eta_{2}} \eta_{1} x \prod_{p \in \mathcal{P}^{c}(2, X)}\left(1-\frac{1}{p}\right)
$$

for all $x \in\left[X^{1-\varepsilon}, 2 X\right]$. This is again possible by Lemma 4.3 when $\eta_{2}<\eta_{5} e^{-2 \lambda /(1-\kappa)}$. 
We consider the set

$$
\mathcal{A}=\left\{m n: n \in \mathcal{N}^{\prime}\left(2 X^{1-\eta_{5}}, X^{1-\eta_{4}}\right), p \mid m \Longrightarrow p \in \mathcal{P}\left(X^{\eta_{2}}, X^{\eta_{5}}\right), m \text { square-free }\right\}
$$

counted with multiplicity. We will show a lower bound for $|\mathcal{A}(X, 2 X)| / X$ and an upper bound for $\left|\mathcal{A}\left(x, x+X^{\varepsilon}\right)\right| / X^{\varepsilon}$ for (almost all) $x \sim X$. The ratio of these bounds will be essentially (16), and the claim follows.

To be able to apply results from the literature directly, we extend $\mathcal{P}\left(X^{\eta_{2}}, X^{\eta_{5}}\right)$ to a set of primes $\mathcal{P}_{e}$ in such a way that (17) holds for all $z \geq w \geq 2$ and $\mathcal{P}_{e}\left(X^{\eta_{2}}, X^{\eta_{5}}\right)=\mathcal{P}\left(X^{\eta_{2}}, X^{\eta_{5}}\right)$. Now (17) implies the normal sieve dimension assumption

$$
\prod_{p \in \mathcal{P}_{e}^{c}(w, z)}\left(1-\frac{1}{p}\right)^{-1}=\left(1+O\left(\frac{1}{\log w}\right)\right)\left(\frac{\log z}{\log w}\right)^{\kappa}
$$

for all $z>w \geq 2$.

In the long interval $[X, 2 X]$, we have

$$
\begin{aligned}
& |\mathcal{A}(X, 2 X)|=(1+o(1)) \sum_{\substack{X^{\eta_{4}} \leq m \leq X^{\eta_{5}} \\
p \mid m \stackrel{p}{\Longrightarrow} \in \mathcal{P}\left(X^{\eta_{2}}, X^{\eta_{5}}\right)}}|\mu(m)| \sum_{n \in \mathcal{N}^{\prime}(X / m, 2 X / m)} 1 \\
& \geq c_{\eta_{2}}(1-o(1)) X \prod_{p \in \mathcal{P}^{c}(2, X)}\left(1-\frac{1}{p}\right) \sum_{\substack{X^{\eta_{4}} \leq m \leq X^{\eta_{5}} \\
p \mid m \stackrel{p}{\Longrightarrow} \in \mathcal{P}\left(X^{\eta_{2}}, X^{\eta_{5}}\right)}} \frac{|\mu(m)|}{m}
\end{aligned}
$$

Next we use a simple trick also present in $\left[4\right.$, Section 2]: For any disjoint $\mathcal{P}_{a}, \mathcal{P}_{b} \subseteq \mathbb{P}$, we have

$$
\sum_{\substack{m \leq X \\ p \mid m \Longrightarrow\left(\mathcal{P}_{a} \cup \mathcal{P}_{b}\right)(2, X)}} \frac{|\mu(m)|}{m} \leq \prod_{p \in \mathcal{P}_{a}(2, X)}\left(1+\frac{1}{p}\right) \sum_{\substack{m \leq X \\ p \mid m \Longrightarrow}} \frac{|\mu(m)|}{m} .
$$

Applying this twice, the last sum in (19) is

$$
\begin{aligned}
& \sum_{\substack{1 \leq m \leq X^{\eta_{5}} \\
p \in \mathcal{P}\left(X^{\eta_{2}}, X^{\eta_{5}}\right)}} \frac{|\mu(m)|}{m}-\sum_{\substack{1 \leq m<X^{\eta_{4}} \\
p \in \in \mathcal{P}\left(X^{\eta_{2}}, X^{\eta_{4}}\right)}} \frac{|\mu(m)|}{m} \\
\geq & \prod_{p \in \mathcal{P}_{e}\left(2, X^{\eta_{2}}\right)}\left(1+\frac{1}{p}\right)^{-1} \sum_{\substack{1 \leq m \leq X^{\eta_{5}} \\
p \mid m \in \mathcal{P}_{e}\left(2, X^{\eta_{5}}\right)}} \frac{|\mu(m)|}{m}-\prod_{p \in \mathcal{P}\left(X^{\eta_{2}}, X^{\eta_{4}}\right)}\left(1+\frac{1}{p}\right) .
\end{aligned}
$$

Since the set $\mathcal{P}_{e}$ is very nicely distributed, we can apply Lemma 4.2 . Writing $\chi_{e}$ for the characteristic function of the set $\mathcal{P}_{e}$, recalling (18) and applying Mertens' formula, the sum over $m$ is

$$
\begin{aligned}
& \geq \frac{\left(\log X^{\eta_{5}}\right)^{1-\kappa}}{\Gamma(1-\kappa+1)} \prod_{p \in \mathbb{P}}\left(1-\frac{1}{p}\right)^{1-\kappa}\left(1+\frac{\chi_{e}(p)}{p}\right)+O(1) \\
& =(1+o(1)) \frac{\left(\log X^{\eta_{5}}\right)^{1-\kappa}}{\Gamma(2-\kappa)}\left(\frac{e^{-\gamma}}{\log X^{\eta_{2}}}\right)^{1-\kappa} \prod_{p \in \mathcal{P}_{e}\left(2, X^{\eta_{2}}\right)}\left(1+\frac{1}{p}\right)+O(1) \\
& =\frac{(1+o(1))}{\Gamma(2-\kappa)}\left(\frac{\eta_{5} e^{-\gamma}}{\eta_{2}}\right)^{1-\kappa} \prod_{p \in \mathcal{P}_{e}\left(2, X^{\eta_{2}}\right)}\left(1+\frac{1}{p}\right)+O(1)
\end{aligned}
$$


Substituting this back to (20) and (19), we get

$$
\frac{|\mathcal{A}(X, 2 X)|}{X} \geq\left(\frac{e^{\gamma \kappa}}{\Gamma(2-\kappa)}-o(1)\right) c_{\eta_{2}} e^{-\gamma}\left(\frac{\eta_{5}}{\eta_{2}}\right)^{1-\kappa} \prod_{p \in \mathcal{P}^{c}(2, X)}\left(1-\frac{1}{p}\right) .
$$

Consider next the short intervals. Let $y=x(2 X)^{\varepsilon-\eta_{1}-1}$ and $y_{1}=x \exp \left(-3(\log X)^{1 / 3}\right)$. We divide $\mathcal{A}$ into subsets according to the range of $n$, that is, for $N \in\left[2 X^{1-\eta_{5}}, \frac{1}{2} X^{1-\eta_{4}}\right]$, we write

$$
\begin{aligned}
\mathcal{A}^{(N)} & =\left\{m n: n \in \mathcal{N}^{\prime}(N, 2 N), p \mid m \Longrightarrow p \in \mathcal{P}\left(X^{\eta_{2}}, X^{\eta_{5}}\right), m \text { square-free }\right\} \\
& \subseteq\left\{m n: n \in \mathcal{N}^{\prime}(N, 2 N),\left(m, \Pi_{1}\right)=\left(m, \Pi_{2}\right)=1\right\},
\end{aligned}
$$

where

$$
\Pi_{1}=\prod_{p \in \mathcal{P}^{c}\left(X^{\eta_{2}}, X^{\left.1-4 \eta_{3} / N\right)}\right.} p \text { and } \Pi_{2}=\prod_{p<X^{\eta_{2}}} p .
$$

We will detect the co-primeness conditions using the sieve weights introduced in Lemma 3.1: Let $\left(\lambda_{d}^{+}\right)$be $\kappa$-dimensional upper bound sieve weights of level $D_{1}=$ $X^{1-3 \eta_{3}} / N$ and let $\left(\widetilde{\lambda}_{d}^{+}\right)$be linear $(\kappa=1)$ upper bound sieve weights of level $D_{2}=$ $X^{\eta_{3}}$. Then using the inclusion above and applying Lemma 3.1(2), we get that

$$
\begin{aligned}
\left|\mathcal{A}^{(N)}(x, x+y)\right| \leq & \sum_{\substack{x \leq m n \leq x+y \\
n \in \mathcal{N}^{\prime}(N, 2 N) \\
\left(m, \Pi_{1}\right)=\left(m, \Pi_{2}\right)=1}} 1 \leq \sum_{\substack{x \leq m n \leq x+y \\
n \in \mathcal{N}^{\prime}(N, 2 N)}} \sum_{d_{1} \mid\left(m, \Pi_{1}\right)} \lambda_{d_{1}}^{+} \sum_{d_{2} \mid\left(m, \Pi_{2}\right)} \tilde{\lambda}_{d_{2}}^{+} \\
= & \sum_{\substack{x \leq d_{1} d_{2} m^{\prime} n \leq x+y \\
n \in \mathcal{N}^{\prime}(N, 2 N), d_{i} \mid \Pi_{i}}} \lambda_{d_{1}}^{+} \widetilde{\lambda}_{d_{2}}^{+} .
\end{aligned}
$$

Adding and substracting $y / y_{1}$ times the same sum over the interval $\left[x, x+y_{1}\right]$, and evaluating the sum over $m^{\prime}$ in the added term we get

$$
\begin{aligned}
\left|\mathcal{A}^{(N)}(x, x+y)\right| \leq & \frac{y}{y_{1}} \sum_{\substack{n \in \mathcal{N}^{\prime}(N, 2 N), d_{i} \mid \Pi_{i}\\
}} \lambda_{d_{1}}^{+} \tilde{\lambda}_{d_{2}}^{+} \cdot\left(\frac{y_{1}}{d_{1} d_{2} n}+O(1)\right) \\
& \sum_{\substack{x \leq d_{1} d_{2} m^{\prime} n \leq x+y \\
n \in \mathcal{N}^{\prime}(N, 2 N), d_{i} \mid \Pi_{i}}}^{+} \tilde{\lambda}_{d_{1}}^{+}-\frac{y}{y_{d_{2}}} \sum_{\substack{x \leq d_{1} d_{2} m^{\prime} n \leq x+y_{1} \\
n \in \mathcal{N}^{\prime}(N, 2 N), d_{i} \mid \Pi_{i}}} \lambda_{d_{1}}^{+} \tilde{\lambda}_{d_{2}}^{+} .
\end{aligned}
$$

The expression in the second line is $O\left(y(\log X)^{-A}\right)$ for almost all $x \sim X$ by Lemma 3.3 with $m=d_{1} d_{2} n$ and $r=m^{\prime} \in\left[X /\left(2 N D_{1} D_{2}\right), 3 X / N\right] \subseteq\left[X^{2 \eta_{3}} / 2,3 X^{\eta_{5}}\right]$. Applying Lemma $3.1(3)$ to the sums over $d_{1}$ and $d_{2}$ in the first line, we get that for almost all $x \sim X,\left|\mathcal{A}^{(N)}(x, x+y)\right|$ is asymptotically at most

$$
\begin{aligned}
& \sum_{n \in \mathcal{N}^{\prime}(N, 2 N)} \frac{y}{n} F_{\kappa}\left(\frac{\log \frac{X^{1-3 \eta_{3}}}{N}}{\log \frac{X^{1-4 \eta_{3}}}{N}}\right) \prod_{p \in \mathcal{P}^{c}\left(X^{\eta_{2}}, X^{\left.1-4 \eta_{3} / N\right)}\right.}\left(1-\frac{1}{p}\right) F_{1}\left(\frac{\eta_{3}}{\eta_{2}}\right) \prod_{p<X^{\eta_{2}}}\left(1-\frac{1}{p}\right) \\
& =y\left(F_{\kappa}(1)+o(1)\right) \prod_{p<X^{\eta_{2}}}\left(1-\frac{1}{p}\right) \sum_{n \in \mathcal{N}^{\prime}(N, 2 N)} \frac{\left(\log X^{\eta_{2}}\right)^{\kappa}}{n(\log X / N)^{\kappa}}
\end{aligned}
$$

by Lemma 3.1(5) and (18).

Summing over $N$ we see that, for almost all $x \sim X$,

$$
|\mathcal{A}(x, x+y)|=y\left(F_{\kappa}(1)+o(1)\right) \frac{e^{-\gamma}}{\left(\log X^{\eta_{2}}\right)^{1-\kappa}} \sum_{n \in \mathcal{N}^{\prime}\left(2 X^{1-\eta_{5}}, X^{1-\eta_{4}}\right)} \frac{1}{n(\log X / n)^{\kappa}} .
$$


Since $\kappa \in(0,1)$, the last sum is by partial summation

$$
\begin{aligned}
& \leq c_{\eta_{2}}(1+o(1)) \prod_{p \in \mathcal{P}^{c}(2, X)}\left(1-\frac{1}{p}\right) \int_{X^{1-\eta_{5}}}^{X^{1-\eta_{4}}} \frac{d t}{t(\log X / t)^{\kappa}} \\
& =\frac{c_{\eta_{2}}(1+o(1))}{(\log X)^{\kappa-1}} \prod_{p \in \mathcal{P}^{c}(2, X)}\left(1-\frac{1}{p}\right) \int_{1-\eta_{5}}^{1-\eta_{4}} \frac{d \beta}{(1-\beta)^{\kappa}} \\
& \leq \frac{c_{\eta_{2}}(1+o(1))}{(\log X)^{\kappa-1}} \prod_{p \in \mathcal{P}^{c}(2, X)}\left(1-\frac{1}{p}\right) \frac{\eta_{5}^{1-\kappa}}{1-\kappa}
\end{aligned}
$$

Hence

$$
|\mathcal{A}(x, x+y)| \leq y\left(\frac{F_{\kappa}(1)}{1-\kappa}+o(1)\right) c_{\eta_{2}} e^{-\gamma}\left(\frac{\eta_{5}}{\eta_{2}}\right)^{1-\kappa} \prod_{p \in \mathcal{P}^{c}(2, X)}\left(1-\frac{1}{p}\right)
$$

for almost all $x \sim X$. On the other hand trivially $|\mathcal{A}(x, x+y)| \ll y$ for all $x \sim X$. Combining these shorter intervals,

$$
\frac{\left|\mathcal{A}\left(x, x+X^{\varepsilon}\right)\right|}{X^{\varepsilon}} \leq\left(\frac{F_{\kappa}(1)}{1-\kappa}+o(1)\right) c_{\eta_{2}} e^{-\gamma}\left(\frac{\eta_{5}}{\eta_{2}}\right)^{1-\kappa} \prod_{p \in \mathcal{P}^{c}(2, X)}\left(1-\frac{1}{p}\right)
$$

for almost all $x \sim X$ and $\left|\mathcal{A}\left(x, x+X^{\varepsilon}\right)\right| \ll X^{\varepsilon}$ for all $x \sim X$.

Let $\beta X$ be the measure of the set of those $x \in[X, 2 X]$ for which $\left|\mathcal{N}\left(x, x+X^{\varepsilon}\right)\right| \geq$ $\eta_{1} X^{\varepsilon} \prod_{p \in \mathcal{P}^{c}(2, X)}\left(1-\frac{1}{p}\right)$. By $(22)$

$$
\begin{aligned}
\frac{|\mathcal{A}(X, 2 X)|}{X} \leq & \beta\left(\frac{F_{\kappa}(1)}{1-\kappa}+o(1)\right) c_{\eta_{2}} e^{-\gamma}\left(\frac{\eta_{5}}{\eta_{2}}\right)^{1-\kappa} \prod_{p \in \mathcal{P}^{c}(2, X)}\left(1-\frac{1}{p}\right) \\
& +\eta_{1} \prod_{p \in \mathcal{P}^{c}(2, X)}\left(1-\frac{1}{p}\right)+O\left((\log X)^{-10}\right) .
\end{aligned}
$$

Combining this with the lower bound (21) implies that

$$
\beta \geq \frac{e^{\gamma \kappa}(1-\kappa)}{F_{\kappa}(1) \Gamma(2-\kappa)}-o(1)=\frac{e^{\gamma \kappa}}{F_{\kappa}(1) \Gamma(1-\kappa)}-o(1) .
$$

For $\kappa=1 / 2$, the previous lemma gives proportion

$$
\frac{e^{\gamma / 2}}{F_{1 / 2}(1) \Gamma(1 / 2)}-\varepsilon^{\prime}=\frac{1}{2}-\varepsilon^{\prime}
$$

which annoyingly just misses what we would like to have (compare with Proposition 2.3 and the discussion thereafter). We are able to overcome this difficulty thanks to the following application of Lemma 3.4.

Lemma 4.7. Let $\sigma \in(0,1), \theta \in(0,1 / 2), \eta_{2}>0$ and $X \rightarrow \infty$. There exists a small positive constant $\eta_{1}=\eta_{1}\left(\sigma, \eta_{2}, \theta\right)$ and a large positive constant $C_{2}=$ $C_{2}\left(\sigma, \eta_{2}, \theta\right)$ such that the following holds for any $B \subseteq \mathbb{N}$. If, for every $X^{\prime} \in$ $\left[X^{1 / 2} / e^{2 \eta_{2}}, e^{2 \eta_{2}} X^{1 / 2}\right]$,

$$
\left|B\left(x, x+X^{\theta}\right)\right| \geq Y(X) X^{\theta}
$$


for proportion $\sigma$ of $x \in\left[X^{\prime}, e^{\eta_{1}} X^{\prime}\right]$, then

$$
\left|\left\{\left(b_{1}, b_{2}\right) \in B \times B: b_{1} b_{2} \in\left[x, x+C_{2} X^{1 / 2+\theta}\right]\right\}\right| \gg_{\sigma, \eta_{2}, \theta} Y(X)^{2} X^{1 / 2+\theta}
$$

for at least proportion $\min \left\{1, \frac{6}{5} \sigma\right\}$ of $x \in\left[X / e^{2 \eta_{2}}, e^{2 \eta_{2}} X\right]$.

Proof. Dividing $\left[X / e^{2 \eta_{2}}, e^{2 \eta_{2}} X\right]$ into subintervals, it is clearly enough to prove the claim in the case $\eta_{2}$ is small. The constant $\eta_{1}$ will be even smaller. Also, besides $C_{2}$, we use another large constant $C_{1}<C_{2}$. In this proof we write $o(1)$ for a quantity which tends to 0 when $C_{1}, C_{2} / C_{1}, \eta_{2} / \eta_{1} \rightarrow \infty$ and $\eta_{2} \rightarrow 0$ (the rate of convergence of $o(1)$ will depend on $\sigma, \theta$ and the original $\eta_{2}$ ). In particular we can assume in the proof that $C_{i}$ are large enough and $\eta_{i}$ are small enough.

For $j \in \mathbb{Z}$, write $x_{j}=X^{1 / 2} \exp \left(j C_{1} X^{\theta-1 / 2}\right)$. We split $\left[X^{1 / 2} / e^{\eta_{2}}, e^{\eta_{2}} X^{1 / 2}\right]$ into intervals $\left[x_{j}, x_{j+1}\right]$ with $j \in[-J, J-1]$, where $J=\eta_{2} X^{1 / 2-\theta} / C_{1}$. The assumption that $\eta_{2}$ is small guarantees that

$$
x_{j+1}-x_{j}=(1+o(1)) C_{1} X^{\theta}
$$

for every $|j| \leq 2 J$. We write

$$
A=\left\{j \in[-J, J-1]:\left|B\left(x_{j}, x_{j+1}\right)\right| \geq Y(X) X^{\theta}\right\} .
$$

By our assumptions

$$
|A| \geq(\sigma-o(1)) 2 J
$$

Notice that if $r_{A+A}(j) \geq \eta_{1} J$, then

$$
\left|\left\{\left(b_{1}, b_{2}\right) \in B \times B: b_{1} b_{2} \in\left[X^{1 / 2} x_{j}, X^{1 / 2} x_{j+2}\right]\right\}\right| \gg J Y(X)^{2} X^{2 \theta}
$$

and thereby

$$
\left|\left\{\left(b_{1}, b_{2}\right) \in B \times B: b_{1} b_{2} \in\left[x, x+5 C_{1} X^{1 / 2+\theta}\right]\right\}\right| \gg Y(X)^{2} X^{1 / 2+\theta}
$$

for every $x \in\left[X^{1 / 2} x_{j-2}, X^{1 / 2} x_{j-1}\right]$.

Hence the claim holds unless

$$
\left|\left\{j \in A+A:: r_{A+A}(j) \geq \eta_{1} J\right\}\right|<\min \left\{1,\left(\frac{6}{5}+\frac{1}{100}\right) \sigma\right\}(4 J-1)
$$

The right hand side is at most $(5 / 2-1 / 50) \sigma \cdot 2 J \leq(5 / 2-3 \cdot 1 / 200)|A|-2$ by $(24)$ since $J$ is large. Now

$$
\eta_{1} J \leq \frac{\eta_{1}}{\sigma^{2}} \cdot \frac{(\sigma \cdot 2 J)^{2}}{4 J} \leq\left(\frac{2 \eta_{1}^{1 / 2}}{\sigma}\right)^{2} \cdot \frac{|A|^{2}}{|2 A|}
$$

by (24), and so by Lemma 3.4 with $\eta=2 \eta_{1}^{1 / 2} / \sigma$ there is an arithmetic progression $P=a_{0}+\left[-a_{1}, a_{2}\right] v \subseteq[-J, J-1]$ such that

$$
|A \cap P| \geq(1-o(1))|A| \quad \text { and } \quad|P| \leq \frac{3}{2}|A| .
$$

Certainly the common difference $v$ is at most $1 / \sigma+o(1)$ and we can force $a_{0} \in$ $[0,1 / \sigma+o(1)]$. By the assumption that $A$ contains many elements from both ends of the interval, $P$ must span over most of the interval, and hence we must have $a_{1}, a_{2} \geq(1-o(1)) J / v=J^{*}$, say.

Since

$$
\left|\left(a_{0}+\left[-J^{*}, J^{*}\right] v\right) \cap A\right| \geq(2 / 3-o(1)) 2 J^{*}
$$


we see by the pigeon hole principle that $r_{A+A}\left(2 a_{0}\right) \gg J$. Furthermore

$$
\left|\left(a_{0}+\left[-I, J^{*}\right] v\right) \cap A\right| \geq\left(\frac{1}{2}+\eta_{1}\right)\left(I+J^{*}\right)
$$

if $I \geq(1 / 3+o(1)) J^{*}$, and the same holds when $\left[-I, J^{*}\right]$ is replaced by $\left[-J^{*}, I\right]$. Hence by the pigeon-hole principle

$$
r_{A+A}\left(2 a_{0}+j^{\prime} v\right) \gg J \text { for every } j^{\prime} \in\left[(-2 / 3+o(1)) J^{*},(2 / 3-o(1)) J^{*}\right],
$$

and so (25) holds for all these $j=2 a_{0}+j^{\prime} v$. Hence

$$
\left|\left\{\left(b_{1}, b_{2}\right) \in B \times B: b_{1} b_{2} \in\left[x, x+\frac{10 C_{1}}{\sigma} X^{1 / 2+\theta}\right]\right\}\right| \gg Y(X)^{2} X^{1+\theta}
$$

for every $x \in\left[X / e^{\eta_{2} / 2}, e^{\eta_{2} / 2} X\right]$.

Repeating the argument for $\left[X / e^{2 \eta_{2}}, X / e^{\eta_{2} / 2}\right]$ and $\left[e^{\eta_{2} / 2} X, e^{2 \eta_{2}} X\right]$, and so forth, we eventually reach the wanted proportion.

That the assumption of lemma must be satisfied for almost all $x \in\left[X^{\prime}, e^{\eta_{1}} X^{\prime}\right]$ for all $X^{\prime} \in\left[X / e^{2 \eta_{2}}, e^{2 \eta_{2}} X\right]$ is not a problem; whenever we can prove the assumption for an interval like $\left[X^{\prime}, 2 X^{\prime}\right]$, we can do it for any $\left[c X^{\prime}, C X^{\prime}\right]$. In particular the interval $[X, 2 X]$ in Lemmas 4.4 and 4.6 can be replaced by $\left[X^{\prime}, e^{\eta_{1}} X^{\prime}\right]$. Combining the previous lemma with Lemma 4.6, we get the following result.

Lemma 4.8. Let $\varepsilon, \varepsilon^{\prime}, \eta_{5}>0, \kappa \in(0,1)$ and $X \rightarrow \infty$. Let $F_{\kappa}(s)$ be as in Lemma 3.1. There exists a small positive constant $\eta_{2}=\eta_{2}\left(\varepsilon, \varepsilon^{\prime}, \eta_{5}, \kappa\right)$ and a constant $C=C\left(\varepsilon, \varepsilon^{\prime}, \eta_{5}, \kappa\right)$ such that if

$$
\sum_{p \in \mathcal{P}^{c}(w, z)} \frac{1}{p} \leq \kappa \sum_{p \in \mathbb{P}(w, z)} \frac{1}{p}
$$

for all $w, z \in\left[X^{\eta_{2}}, X^{\eta_{5}}\right]$ with $z>\left(1+\eta_{2}\right) w$, then

$$
\mathcal{N}\left(\left[x, x+C X^{1 / 2+\varepsilon}\right]\right) \gg_{\varepsilon, \varepsilon^{\prime}, \eta_{5}, \kappa} \frac{X^{1 / 2+\varepsilon}}{\max _{n \in \mathcal{N}(X, 3 X)} \tau(n)} \prod_{p \in \mathcal{P}^{c}(2, X)}\left(1-\frac{1}{p}\right)^{2}
$$

for at least proportion

$$
\min \left\{1, \frac{6}{5} \cdot \frac{e^{\gamma \kappa}}{F_{\kappa}(1) \Gamma(1-\kappa)}-\varepsilon^{\prime}\right\}
$$

of $x \in[X, 2 X]$.

\section{Proofs of Theorems 1.1-1.3}

5.1. Almost all forms - proof of Theorem 1.3. We first prove Theorem 1.3 as its reduction from results in the previous section is easiest. We need the following lemma in the reduction.

Lemma 5.1. Let $0<\beta_{1}<\beta_{2} \leq 1 / 25$. Then

$$
\sum_{\substack{k^{\beta_{1}} \leq p \leq k^{\beta_{2}} \\\left|\lambda_{f}(p)\right| \geq 1 / 5}} \frac{1}{p} \geq\left(\frac{1}{2}+\frac{1}{24}\right) \sum_{\substack{k_{1}^{\beta} \leq p \leq k^{\beta_{2}}\\}} \frac{1}{p}+O\left(k^{-\beta_{1} / 5}\right)
$$

for all $f \in H_{k}$ with $\ll k^{1-\beta_{1} / 2}$ exceptions. 
Proof. This follows as [11, Lemma 4.1] but using the large sieve inequality (Lemma 3.2 ) instead of holomorphy and non-vanishing at $s=1$ of the second, fourth and sixth symmetric power $L$-functions. The main point of the proof is existence of a polynomial

such that

$$
Y(x)=\frac{1}{2}+\frac{1}{24}+\alpha_{2} U_{2}(x)+\alpha_{4} U_{4}(x)+\alpha_{6} U_{6}(x)
$$

$$
Y(x) \leq \begin{cases}0 & \text { if }|x|<1 / 5 \\ 1 & \text { if }|x| \in[1 / 5,2]\end{cases}
$$

Here $U_{j}(x)$ is the $j$ th Chebyshev polynomial, so that $U_{j}\left(\lambda_{f}(p)\right)=\lambda_{f}\left(p^{j}\right)$.

Remark 5.2. One could get much better numbers than $1 / 2+1 / 24$ and $1 / 5$ in the previous lemma by using larger powers $\lambda_{f}\left(p^{j}\right)$-indeed one could approach the Sato-Tate distribution when $\beta_{2} \rightarrow 0$. However this lemma suffices to our needs. Also one could prove a similar result for smaller $p$, which could be used to prove a slightly stronger form of Theorem 1.3.

Proof of Theorem 1.3. We can assume that $\varepsilon<1 / 25$. Let $\lambda$ be as in Lemma 4.3 and let $\eta=\varepsilon e^{-2 \lambda}, Y=k^{1 / 2} \log k, X=k /(20 Y)$, and let $\mathcal{P}_{1}$ and $\mathcal{N}_{1}$ be as in (5). By the previous lemma

$$
\sum_{p \in \mathcal{P}_{1}\left(X^{\eta}, X^{\varepsilon}\right)} \frac{1}{p} \geq\left(\frac{1}{2}+\frac{1}{30}\right) \sum_{p \in \mathbb{P}\left(X^{\eta}, X^{\varepsilon}\right)} \frac{1}{p} \geq \lambda
$$

for all $f \in H_{k}$ with $\ll k^{1-\eta / 2}$ exceptions.

By feeding this into Lemma 4.4 , we see that, for all but $\ll k^{1-\eta / 2}$ cusp forms $f \in H_{k}$, one has, for both $\nu=1$ and for $\nu=\omega_{f}$,

$$
\mathcal{N}_{1}\left(x / \nu,\left(x+X^{\varepsilon}\right) / \nu\right) \gg \frac{X^{\varepsilon}}{\log X}
$$

for almost all $x \sim X$. Consequently by Proposition 2.3(i)

$$
\left|\mathcal{Z}(f) \cap\left(\delta_{1} \cup \delta_{2}\right)\right| \gg X^{1-\varepsilon} \gg\left(\frac{k^{1 / 2}}{\log k}\right)^{1-\varepsilon} \gg k^{1 / 2-2 \varepsilon / 3}
$$

for all but $\ll k^{1-\eta / 2}$ cusp forms.

5.2. All forms - proof of Theorem 1.1. We will give a bit more precise information about location of zeros than Theorem 1.1 and consider $\mathcal{F}_{Y}$ (defined in (3)) with $Y$ in the range

$$
\sqrt{k \log k} \ll Y<\frac{1}{100} k .
$$

Ghosh and Sarnak [3, Theorem 1.2] showed that in this range

and

$$
\left|\mathcal{Z}(f) \cap \mathcal{F}_{Y}\right| \asymp \frac{k}{Y}
$$

$$
\left|\mathcal{Z}(f) \cap \mathcal{F}_{Y} \cap\left(\delta_{1} \cup \delta_{2}\right)\right| \gg_{\varepsilon}\left(\frac{k}{Y}\right)^{1 / 2-1 / 40-\varepsilon} .
$$

Here we remove the fraction 1/40 which was done in [3] only under the Riemann hypothesis. 
Theorem 5.3. Let $\varepsilon>0$ and $k \rightarrow \infty$. If (27) holds, then

$$
\left|\mathcal{Z}(f) \cap \mathcal{F}_{Y} \cap\left(\delta_{1} \cup \delta_{2}\right)\right| \gg_{\varepsilon}\left(\frac{k}{Y}\right)^{1 / 2-\varepsilon} .
$$

Proof. We can assume that $Y$ satisfies (4) - for smaller $Y$ the claim follows from the claim for $Y=\beta_{1}^{\prime} \sqrt{k \log k}$ and for larger $Y$ the claim follows from the result of Ghosh and Sarnak. Also we can clearly assume that $\varepsilon$ is small. Choose $\kappa>1 / 2$ such that

$$
\frac{6}{5} \cdot \frac{e^{\kappa \gamma}}{F_{\kappa}(1) \Gamma(1-\kappa)}>\frac{1}{2}+\frac{1}{100} .
$$

Such $\kappa$ exists by continuity since for $\kappa=1 / 2$ the left hand side is $3 / 5>1 / 2+1 / 100$. Let $\eta_{2}$ be the constant got from Lemma 4.8 with $\kappa$ and $\varepsilon$ as here, $\varepsilon^{\prime}=1 / 1000$ and $\eta_{5}=\varepsilon$. Let $\eta=\min \left\{\eta_{2}, e^{-5 \lambda} \varepsilon\right\}$, where $\lambda$ is as in Lemma 4.3.

Let $X=k /(20 Y)$ and consider the sets $\mathcal{P}_{1}, \mathcal{P}_{2}, \mathcal{N}_{1}$ and $\mathcal{N}_{2}$ introduced in (5). Recalling that $\mathcal{P}_{1} \cup \mathcal{P}_{2}=\mathbb{P}\left(X^{\eta}, X\right)$ we split into three cases.

Case 1: $\mathcal{P}_{2}$ satisfies the assumptions of Lemma 4.4 with $X, \eta$ and $\varepsilon$ there $X^{1 / 2}, 2 \eta$ and $2 \varepsilon$ here and $\delta=\kappa-1 / 2$.

By Lemma 4.4, we have, for both $\nu=1$ and $\nu=\omega_{f}$,

$$
\mathcal{N}_{2}\left(x / \nu^{1 / 2},\left(x+X^{\varepsilon}\right) / \nu^{1 / 2}\right) \gg \frac{X^{\varepsilon}}{(\log X)^{3}}
$$

for almost all $x \sim X^{1 / 2}$, and the claim follows from Proposition 2.3(ii).

Case 2: $\mathcal{P}_{1}$ satisfies the assumptions of Lemma 4.8 with $X, \varepsilon, \varepsilon^{\prime}, \eta_{2}, \eta_{5}$ and $\kappa$ as here.

Recalling that $\eta_{2}$ was chosen to be the parameter in Lemma 4.8, we can apply that lemma to get that, by (28), for proportion at least $1 / 2+1 / 200$ of $x \sim X$, one has $\mathcal{N}_{1}\left(x, x+C X^{1 / 2+\varepsilon}\right) \gg X^{1 / 2+\varepsilon} /(\log X)^{2}$. The same holds for $\mathcal{N}_{1}\left(x / \omega_{f},(x+\right.$ $\left.\left.C X^{1 / 2+\varepsilon}\right) / \omega_{f}\right)$ and the claim follows from Proposition 2.3(i).

Case 3: The remaining case.

Since $\mathcal{P}_{1} \cup \mathcal{P}_{2}=\mathbb{P}\left(X^{\eta}, X\right)$, the condition (11) for $\mathcal{P}_{2}$ and the condition (26) for $\mathcal{P}_{1}$ are complementary. Hence if we are not in cases 1 or 2 , then

$$
\sum_{p \in \mathcal{P}_{2}\left(X^{\eta}, X^{1 / 2-2 \varepsilon}\right)} \frac{1}{p} \leq \lambda .
$$

Since $\eta \leq e^{-5 \lambda} \varepsilon$, this implies that

$$
\begin{aligned}
\sum_{p \in \mathcal{P}_{1}\left(X^{\eta}, X^{\varepsilon}\right)} \frac{1}{p} & \geq \sum_{p \in \mathbb{P}\left(X^{\eta}, X^{\varepsilon}\right)} \frac{1}{p}-\lambda \geq\left(\frac{1}{2}+\frac{1}{4}\right) \sum_{p \in \mathbb{P}\left(X^{\eta}, X^{\varepsilon}\right)} \frac{1}{p}+\frac{1}{4} \log \frac{\varepsilon}{\eta}-o(1)-\lambda \\
& \geq\left(\frac{1}{2}+\frac{1}{4}\right) \sum_{p \in \mathbb{P}\left(X^{\eta}, X^{\varepsilon}\right)} \frac{1}{p} \geq \lambda .
\end{aligned}
$$

Hence $\mathcal{P}_{1}$ satisfies the assumptions of Lemma 4.4 with $\delta=1 / 4$ and $X, \eta, \varepsilon$ as here, so, for $\nu=1$ and $\nu=\omega_{f}$,

$$
\mathcal{N}_{1}\left(x / \nu,\left(x+X^{\varepsilon}\right) / \nu\right) \gg \frac{X^{\varepsilon}}{\log X}
$$


for almost all $x \sim X$. Hence in this case Proposition 2.3(i) gives even

$$
\left|\mathcal{Z}(f) \cap \mathcal{F}_{Y} \cap\left(\delta_{1} \cup \delta_{2}\right)\right| \gg_{\varepsilon}\left(\frac{k}{Y}\right)^{1-\varepsilon} .
$$

Remark 5.4. Improving Theorem 5.3 significantly seems difficult at least in this $Y$ range. This is because we cannot rule out the possibility that $\left|\lambda_{f}(p)\right|$ is extremely small for all primes $p \leq k^{1 / 2}$, in which case (by the proof of our Proposition 2.1 in [3] $), f(\alpha+i y)$ would be dominated by a multiple of $(-1)^{\omega_{f}(l)} e\left(\alpha l^{2}\right)$ for $y$ in long intervals, and there indeed would not be many real zeros with $y>k^{1 / 2} \log k$. On the other hand, existence of many real zeros with $y>k^{1 / 2} \log k$ would provide information about the Fourier coefficients. It is worth mentioning that Serre [18] has shown that $\lambda_{f}(p)$ cannot be exactly 0 often, but since the proof uses algebraic methods it cannot be extended to ruling out small values.

5.3. Zeros on both lines-proof of Theorem 1.2. Below we prove a result which depends on $\hat{n}$, the smallest prime power such that $\lambda_{f}(\hat{n}) \leq-\varepsilon_{0}$ for some constant $\varepsilon_{0}$. Theorem 1.2 follows since Ghosh and Sarnak [3, Proposition 4.4] showed that the arguments in [11] and [14] on smallest negative eigenvalue together with $k$-aspect subconvexity bounds for $L(s, f)$ from [15] and [10] yield $\hat{n} \ll k^{0.4963}$.

Theorem 5.5. Let $\varepsilon>0, k \rightarrow \infty$ and $f \in H_{k}$. Then

$$
\left|\mathcal{Z}(f) \cap \delta_{2}\right| \gg_{\varepsilon} k^{1 / 8-\varepsilon} \text {. }
$$

Let further $\varepsilon_{0}>0$ and let $\hat{n}$ be the smallest prime power such that $\lambda_{f}(\hat{n}) \leq-\varepsilon_{0}$. If $\hat{n} \leq k^{1 / 2-\varepsilon}$, then

$$
\left|\mathcal{Z}(f) \cap \delta_{1}\right| \gg_{\varepsilon, \varepsilon_{0}} \min \left\{k^{1 / 2-\varepsilon} / \hat{n}, k^{1 / 8-\varepsilon}\right\} .
$$

Proof. Let $Y=k^{1 / 2} \log k, X=k /(20 Y)$ and let $\rho$ be such that $\hat{n}=k^{\rho}$. We first prove the claim concerning $\delta_{1}$. We can clearly assume that $\varepsilon<1 / 2-\rho$ and $\varepsilon_{0}<1 / 10$. Assume first that $\rho>\varepsilon / 4$. By (1) we must have $\lambda_{f}(p) \geq 1 / 2$ for every $p<k^{\rho / 2}$. Let $\lambda$ be as in Lemma 4.3 and $\eta=e^{-8 \lambda} \varepsilon$. Let $\theta=2(\rho+\varepsilon)$. By Lemma 3.3, for almost all $x \sim X$, the interval $\left[x, x+X^{\theta}\right]$ contains products of $\asymp 1 / \rho$ distinct odd primes all of which are smaller than $k^{\rho / 2}$. Let $l_{2}^{\prime}$ be such product, so that $\lambda_{f}\left(l_{2}^{\prime}\right) \geq(1 / 2)^{O(1 / \rho)}$. Now $\frac{X^{\theta}}{\hat{n}}=X^{2(\rho+\varepsilon)} / k^{\rho} \geq X^{\varepsilon}$, so similarly Lemma 3.3 implies that, for almost all $x \sim X$, the interval $\left[x / \hat{n},\left(x+X^{\theta}\right) / \hat{n}\right]$ contains $l_{1}^{\prime}$ such that $\lambda_{f}\left(l_{1}^{\prime}\right) \geq(1 / 2)^{O(1 / \rho)}$. The claim follows from Proposition 2.2(i) for intervals $\left[x, x+X^{\theta}\right]$ with $l_{1}=\hat{n} l_{1}^{\prime}$ and $l_{2}=l_{2}^{\prime}$.

On the other hand, if $\rho \leq \varepsilon / 4$, we can argue as in Section 5.2 to show that, for any constants $C_{2}>C_{1}>0$, for proportion at least $1 / 2+1 / 200$ of $x \in$ $\left[C_{1} X^{1 / 2}, C_{2} X^{1 / 2}\right]$, the interval $\left[x, x+C X^{1 / 4+\varepsilon}\right]$ contains co-prime odd numbers $n_{1}, n_{2}, n_{3}$ with $\left|\lambda_{f}\left(n_{i}\right)\right| \gg 1$. Then multiplying two of these with same signs, we see that, for proportion at least $1 / 2+1 / 300$ of $x \sim X$, the interval $\left[x, x+3 C X^{3 / 4+\varepsilon}\right]$ contains a number $l_{2}^{\prime}$ such that $\lambda_{f}\left(l_{2}^{\prime}\right) \gg 1$. Similarly for proportion at least $1 / 2+1 / 300$ of $x \sim X$ the interval $\left[x / \hat{n},\left(x+3 C X^{3 / 4+\varepsilon}\right) / \hat{n}\right]$ contains numbers $l_{1}^{\prime}$ with $\lambda_{f}\left(l_{1}^{\prime}\right) \gg 1$. The claim follows from Proposition 2.2 (i) for intervals $\left[x, x+3 C X^{3 / 4+\varepsilon}\right]$ with $l_{1}=\hat{n} l_{1}^{\prime}$ and $l_{2}=l_{2}^{\prime}$.

This finishes the proof for $\delta_{1}$ and we can turn to $\delta_{2}$. By [3, Lemma 4.1], there is $b \ll 1$ such that $\lambda_{f}\left(2^{b}\right) \geq 1 / 10$. Now we can use the argument used above in the 
case $\rho \leq \varepsilon / 4$ with $2^{b}$ in the place of $\hat{n}$ to find $l_{1}$ and $l_{2}$ with different parity such that $\lambda_{f}\left(l_{1}\right), \lambda_{f}\left(l_{2}\right) \gg 1$, and the claim again follows from Proposition 2.2(i).

There are some chances to improve the exponent slightly - from proof of $\hat{n} \ll$ $k^{0.4963}$ we actually know that there must be more than one $n \ll k^{0.4963}$ with $\lambda_{f}(n) \leq-\varepsilon_{0}$ which might be advantageous. However, the improvement would probably not be that great since the proof does not guarantee very many of them - one could just have something of sort $\lambda_{f}\left(p^{10}\right)<-\varepsilon_{0}$ for $p^{10} \in\left[k^{0.496}, k^{0.497}\right]$. Due to this weak knowledge about negative eigenvalues it seems difficult to improve the exponent significantly.

\section{ACKNOWLEDGEMENTS}

The author wishes to thank Amit Ghosh and Peter Sarnak for encouragement and the referee for thorough reading of the paper. She is also grateful to her coauthors in [4] as many ideas here, in particular bringing in additive combinatorics, arose from that work.

\section{REFERENCES}

[1] E. Croot. Smooth numbers in short intervals. Int. J. Number Theory, 3:159-169, 2007.

[2] J. B. Friedlander and H. Iwaniec. Opera de Cribro, volume 57 of American Mathematical Society Colloquium Publications. American Mathematical Society, Providence, Rhode Island, 2010.

[3] A. Ghosh and P. Sarnak. Real zeros of holomorphic Hecke cusp forms. J. Eur. Math. Soc., 14:465-487, 2012.

[4] A. Granville, D. Koukoulopoulos, and K. Matomäki. When the sieve works. Preprint, available at http://arxiv.org as arXiv:1205.0413v1 [math.NT].

[5] G. Harman. Prime-detecting Sieves, volume 33 of London Mathematical Society Monographs (New Series). Princeton University Press, Princeton, 2007.

[6] G. Harman and K. Matomäki. Some problems of analytic number theory on arithmetic semigroups. Funct. Approx. Comment. Math., 38:21-40, 2008.

[7] R. Holowinsky and K. Soundararajan. Mass equidistribution for Hecke eigenforms. Ann. of Math. (2), 172(2):1517-1528, 2010.

[8] H. Iwaniec and P. Sarnak. $L^{\infty}$ norms of eigenfunctions of arithmetic surfaces. Ann. of Math. (2), 141(2):301-320, 1995.

[9] C. Jia. Almost all short intervals containing prime numbers. Acta Arith., 76:21-84, 1996.

[10] M. Jutila and Y. Motohashi. Uniform bound for Hecke L-functions. Acta Math., 195:61-115, 2005.

[11] E. Kowalski, Y.-K. Lau, K. Soundararajan, and J. Wu. On modular signs. Math. Proc. Cambridge Philos. Soc., 149(3):389-411, 2010.

[12] Y.-K. Lau and J. Wu. A large sieve inequality of Elliott-Montgomery-Vaughan type for automorphic forms and two applications. Int. Math. Res. Not. IMRN, 5:Art. ID rnm 162, 35, 2008.

[13] V. F. Lev. Restricted set addition in groups. III. Integer sumsets with generic restrictions. Period. Math. Hungar., 42(1-2):89-98, 2001.

[14] K. Matomäki. On signs of Fourier coefficients of cusp forms. Math. Proc. Cambridge Philos. Soc., 152:207-222, 2012.

[15] Z. Peng. Zeros and central values of automorphic L-functions. PhD thesis, Princeton University, 2001.

[16] F. K. C. Rankin and H. P. F. Swinnerton-Dyer. On the zeros of Eisenstein series. Bull. London Math. Soc., 2:169-170, 1970.

[17] Z. Rudnick. On the asymptotic distribution of zeros of modular forms. Int. Math. Res. Not., 34:2059-2074, 2005.

[18] J.-P. Serre. Quelques applications du théorème de densité de Chebotarev. Inst. Hautes Études Sci. Publ. Math., 54:323-401, 1981. 
[19] T. Tao and V. H. Vu. Additive combinatorics, volume 105 of Cambridge Studies in Advanced Mathematics. Cambridge University Press, Cambridge, paperback edition, 2010.

[20] E. Wirsing. Über die Zahlen, deren Primteiler einer gegebenen Menge angehören. Arch. Math., $7: 263-272,1956$

E-mail address: ksmato@utu.fi

Department of Mathematics and Statistics, University of Turku, 20014 Turku, FinLAND 